\title{
Reducción agresiva de los lípidos o angioplastia en angina crónica. El beneficio de la duda
}

Pitt B, Waters D, Virgil Brown W, et al, for the Atorvastatin versus Revascularization Treatment Investigators. Agressive lipid lowering therapy compared with angioplasty in stable coronary artery disease. NEngl J Med 1999;341: 70-6

\section{Objetivo}

Comparar los efectos de una reducción agresiva de los lípidos con la angioplastia sobre la tasa de eventos coronarios y la clase funcional de la angina en pacientes con angina crónica estable.

\section{Diseño}

Ensayo clínico, abierto, randomizado y multicéntrico.

\section{Lugar}

Participaron 37 centros en Estados Unidos y Europa

\section{Pacientes}

Se incluyeron 341 pacientes con angina crónica estable en clase funcional I, II o asintomáticos. Debían cumplir por lo menos 4 minutos de una prueba ergométrica con protocolo de Bruce o cualquier prueba funcional sín signos marcados de isquemia. Eran excluidos los pacientes con enfermedad de tres vasos, compromiso del tronco de la coronaria izquierda, angina inestable o infarto de miocardio reciente y fracción de eyección menor al $40 \%$.

\section{Intervención}

Los pacientes se estratificaron según tuvieran enfermedad de uno o dos vasos, y luego se randomizaron a recibir tratamiento médico con $80 \mathrm{mg}$. de atorvastatina por día o angioplastia seguida de tratamiento médico usual que podía incluir hipolipemiantes. Se asignaron 164 pacientes al grupo atorvastatina y 177 al grupo angioplastia.

\section{Medición de los resultados}

El resultado principal a medir fue la proporción de eventos isquémicos en cada grupo. Se definían los eventos isquémicos como la presencia de por lo menos uno de los siguientes: muerte de causa cardiovascular, resucitación luego de un paro cardíaco, infarto de miocardio no fatal, accidente cerebrovascular, cirugía de revascularización miocárdica, angioplastia o empeoramiento de la angina de pecho con requerimiento de internación.

\section{Resultados}

De la estratificación inicial surgió por azar que el grupo asignado a atorvastatina recibía significativamente menos aspirina, incluía menos hombres, y tenían significativamente más lesiones en la descendente anterior. De cualquier manera la tendencia observada en estos grupos analizados separadamente, mostró ser similar a la de los resultados globales. Del grupo atorvastatina , 22 pacientes (13\%) tuvieron un evento isquémico y lograron una reducción del $46 \%$ del LDL inicial, llegando a una media de $77 \mathrm{mg} / \mathrm{dl}$, comparado con 37 eventos en el grupo angioplastia (21\%), que tuvieron una reducción del $18 \%$ del LDL con una media de $119 \mathrm{mg} / \mathrm{dl}$. La reducción relativa de eventos fue de $36 \%$ luego de un seguimiento de 18 meses $(p=0,048)$. Además, el grupo atorvastatina tuvo una reducción del riesgo al tiempo de un primer evento isquémico del $36 \%$ [IC $95 \%=5$ a $67 \%, p=0,03]$. En el grupo atorvastatina la clase funcional de la angina mejoró en el $41 \%$ de los pacientes, $48 \%$ permaneció estable y $12 \%$ sufrió deterioro; mientras que en el grupo angioplastia mejoró un $54 \%$, permaneció estable un $40 \%$ y empeoró un $7 \%$. Esta diferencia favoreció significativamente a la angioplastia.

\section{Conclusiones}

En pacientes con angina crónica de bajo riesgo, la reducción de eventos isquémicos en el grupo tratado con hipolipemiantes con agresiva reducción de los lípidos fue al menos similar a la observada en el grupo angioplastia seguida de tratamiento usual.

\section{COMENTARIO}

En la angina crónica estable existen dos tipos de tratamiento, el tratamiento médico y los procedimientos de revascularización (cirugía de revascularización y angioplastia). La opción por uno u otro tratamiento depende de variables clínicas y anatómicas, esto último en el caso que se haya decidido realizar una cinecoronariografía (CCG). La cirugía de revascularización (CRM) ha demostrado reducir la mortalidad en los pacientes con compromiso del tronco de la coronaria izquierda, enfermedad de tres vasos 0 en aquellos con dos vasos y deterioro de la función ventricular ${ }^{1}$. Al margen de esta indicación, los procedimientos de revascularización no lograron demostrar reducir la mortalidad ni la aparición de eventos coronarios, aunque si han logrado mejorar la clase funcional de la angina ${ }^{2}$. La elección de un tratamiento u otro depende de la clase funcional de la angina y de su repercusión sobre la calidad de vida del paciente, y/o de la presencia de pruebas funcionales de riesgo, que generalmente están relacionados con el grado de angina como marcador de isquemia. Los procedimientos de revascularización están indicados en pacientes con angina en clase funcional III-IV y en aquellos con clase funcional I-II, en los que la calidad de vida está deteriorada ${ }^{1}$.

El presente estudio parte de la idea que la reducción agresiva del colesterol disminuye los eventos coronarios en mayor medida que la angioplastia. Sin embargo la evidencia disponible muestra que en los pacientes de bajo riesgo la angioplastia no disminuye los eventos coronarios, ${ }^{3-4}$ por lo cual el resultado del estudio no resulta una sorpresa. Los participantes inicialmente no tenían indicación de angioplastia, ya que tenían angina en clase funcional I-II o estaban asintomáticos, y aquellos con pruebas funcionales

de riesgo eran excluidos. Por lo tanto, no resulta biológicamente muy sensato comparar tratamiento agresivo con atorvastatina con angioplastia para prevenir el empeoramiento del angor.

Por otra parte, los resultados del estudio son poco precisos. La reducción relativa de eventos del $36 \%$ reportada en el estudio tiene un IC 95\% estimado entre $2,9 \%$ y $54 \%$, y una reducción absoluta de $7,4 \%(13,4 \%$ en el grupo atorvastatin vs $21 \%$ en el grupo angioplastia) con un IC95\% que va desde $0,4 \%$ al 15,4\%*. Esta dispersión probablemente se debe a que los autores estimaron una tasa de eventos en cada grupo muy superior a la que se observó en el estudio, lo que indudablemente ocasiona una pérdida de poder muy importante.

El estudio para evaluar la intervención debería haber comparado la reducción agresiva de los lípidos con el tratamiento convencional. Otro aspecto cuestionable es que definen como eventos isquémicos distintos cuadros que si bien están relacionados, son en muchos aspectos clínica y fisiopatológicamente distintos, por lo que terminan en un conjunto heterogéneo de eventos que analizados en detalle solo permiten destacar como evento particular el empeoramiento de la angina.

En conclusión no es prudente concluir que reducir agresivamente el colesterol en pacientes con angina crónica y bajo riesgo sea por lo menos igual que la angioplastia ya que de por sí este útimo tratamiento no está indicado en estos pacientes. El aporte del estudio es reafirmar que en pacientes de bajo riesgo el tratamiento médico no es superado por los procedimientos de revascularización y resulta interesante para considerar el efecto de estas drogas sobre la reducción de la angina inestable.

Dr. Augusto Granel

Referencias

Unidad de Medicina Familiar y Preventiva. Hospital Italiano de Buenos Aires.

1. Sociedad Argentina de Cardiología, Asociación Argentina de Angiología y Cirugía Cardiovascula. Consenso de cardiopatía isquémica. Revista Argentina de Cardiología. 65 , supl. II 1997

2. A report of the American Heart Association/ American College of Cardiology, of the Cardiology Task Force (comitee on PTCA). Guidelinees for percutaneous transluminal coronary angioplasty. Circulation, $1993 ; 88,2987-300$

3. Parisi AF, Folland ED, Hartigan P. A comparison of angioplasty with medical therapy in the treatment of single vessel coronary artery disease. N. Engl. J. Med., $1992 ; 326$ :10-16 4. RTA-2 Trial Participants. Coronary angioplasty versus medical therapy for angina : the second randomized intervention treatment of ancina. Lancet $1997: 350: 461-468$ 